\title{
EVALUACIÓN DE LOS COSTOS DE VEHÍCULOS DE PROPULSIÓN ELÉCTRICA Y A GASOLINA
}

\author{
Juan P. E. Gomez \\ Universidad de Brasília (UnB), Programa de Posgrado en Transportes-PPGT \\ Ivo Almeida \\ Empresa Brasilera de Correios e Telégrafos (CORREIOS) \\ Roberto Bernardo da Silva \\ Universidad de Brasília (UnB), Programa de Posgrado en Transportes-PPGT \\ Fabiana S. de Arruda \\ Universidad de Brasília (UnB), Programa de Posgrado en Transportes-PPGT \\ Augusto César de Mendoça Brasil \\ Universidad de Brasília (UnB), Programa de Posgrado en Transportes-PPGT
}

\section{RESUMEN}

El inminente agotamiento de las fuentes de energía primaria, principalmente por la dependencia energética del petróleo trae como resultado la inversión en nuevos modelos de energía para la movilidad destinados a mejorar la calidad de vida de la población, buscando nuevas formas de reducir el uso de combustibles fósiles como fuente de energía. El sistema de transporte ha sufrido muchas críticas por ser responsable del consumo de gran parte de estos combustibles, por lo cual, desde hace algunas décadas se está incentivando a la reducción del transporte individual y uso de vehículos no motorizados, además del uso de fuentes alternativas de energía que incluyen vehículos propulsados por energías renovables. Europa emerge en la integración de los Vehículos Eléctricos (VE) en su flota con el fin de disminuir las emisiones de Gases Efecto Invernadero (GEI). Ya en Brasil, esta tecnología aún se encuentra tímida debido a las restricciones al uso del VE, la principal, sus costos marginales. El objetivo de esta investigación consiste en comparar los costos marginales de dos vehículos con sistemas de propulsión diferentes (gasolina y energía eléctrica). El resultado del estudio permitió analizar los pros y los contras de introducir el VE en Brasil. El método adoptado se divide en dos etapas, la primera considera una revisión bibliográfica mundial y local, seguido del análisis del costo marginal, teniendo en cuenta valores relacionados con los costos de adquisición, mantenimiento e inspección en un período de 100 mil kilómetros, además de la autonomía y valores de impuestos. Los resultados de este estudio mostraron que, incluso si se adoptaran políticas de incentivos en Brasil para el uso del VE en el corto plazo, éstos podrían convertirse en inviable si no se contemplan los componentes más caros. Por lo tanto, el uso intensivo del VE en Brasil es todavía una realidad lejana.

\section{INTRODUCCIÓN}

Los problemas ambientales han sido el orden del día de varias reuniones, debates y seminarios, como una intención de mitigar y de aportar soluciones rápidas y eficientes a este inconveniente. El sector del transporte se ha convertido en el que más ha recibido aporte de ideas y estudios, incluyendo la aparición de vehículos híbridos y eléctricos, además de mejoras en la eficiencia de los motores de combustión interna y la calidad del propio combustible con adición de etanol y biocombustibles. 
Europa emerge en la integración de los vehículos eléctricos en su flota con el fin de disminuir las emisiones de gases de efecto invernadero. En Brasil, la tecnología es aún tímida, porque hay muchas restricciones al uso de vehículos eléctricos, entre las cuales se pueden resaltar, los costos marginales de vehículo eléctrico y la falta de incentivos para su adquisición.

El objetivo de este estudio fue comparar dos combustibles diferentes y la autonomía de los modelos VolksWagen Gol y Nissan Leaf y cuál de estos presenta un mejor costo marginal para así hacer un paralelo con las políticas de incentivo al uso del vehículo eléctrico. Inicialmente se presentará un marco teórico para vehículos eléctricos y de combustión interna, seguido de algunas ventajas y desventajas de cada uno y los incentivos que algunos países han adoptado con respecto al uso de los vehículos eléctricos, finalmente, la aplicación de la metodología propuesta y la presentación de resultados y discusiones generadas.

\section{TRANSPORTE}

Conforme Santos et al. (2012), los transportes se han convertido en el principal medios de locomoción utilizado por los individuos, facilitando el acceso a cualquier lugar con mayor eficiencia y rapidez y día a día gana más importancia en la sociedad. Sin embargo, con el paso del tiempo y gracias al crecimiento poblacional y por consiguiente el tráfico vehicular, el transporte se ha convertido en un problema al convertirse en el principal foco de diversos problemas de contaminación y otros derivados graves problemas de salud.

La palabra "transporte" viene del latín trans (de aquí para allá) y portare (carga). Se puede decir entonces que, en resumen, el transporte es el movimiento de personas o cosas de un lugar a otro. El transporte puede ser distinguido por la posesión, donde el transporte público está dirigido a cualquier persona y privado está restringido a las personas que lo adquieren.

Transporte contiene tres elementos: infraestructura, vehículos y operaciones comerciales. La infraestructura corresponde a la red de transporte, es decir a la carretera, ferrocarril, espacio aéreo y fluvial, etc. Los vehículos son automóviles, bicicletas, autobuses, trenes y aviones, que utilizan esta red. Las operaciones son las formas en que estos vehículos utilizan la red, como las leyes, directrices, códigos, etc. En este artículo, los medios de transporte se pueden dividir en las siguientes categorías: carretera (carro, autobús y tren), agua (buques, canoas y barcos) y aire (aviones, helicópteros y globo).

Por otra parte, en estos días no sólo basta con realizar una buena gestión de la flota, el mercado de servicios de transporte exige a las empresas una modernización constante y uso de nuevas tecnologías ambientalmente eficientes con el fin de mantener o ampliar su cuota de mercado y proteger el medio ambiente. Los avances tecnológicos que corren a grandes velocidades en el mundo, deben ser acompañados de cerca por las empresas y ser implementados siempre que haya la viabilidad técnica y económica. En ese aspecto, Santos, et al., (2012), resaltó que el sector de transportes es la base para la estabilidad de cualquier economía, además de ser indispensable para garantizar la competitividad de un mercado globalizado.

\section{INNOVACION}

De acuerdo con Lounsbury y Crumley (2007), la innovación puede surgir a partir de un proceso de diferentes fases como: identificación de un problema específico, aumento de las dificultades existentes, percepción de una necesidad de prácticas innovadoras y, finalmente, todos los procesos políticos que envuelven la aceptación de determinada innovación por los agentes 
involucrados en el proceso.

Según Gremaud et al. (2004), los procesos de innovación son cada vez más complejos debido principalmente a la aplicación de las nuevas tecnologías y a la reducción progresiva de la gestión del ciclo de vida de un producto, creando así la necesidad de nuevas estrategias de mercado.

Durand (1997), afirma que hay intensidades de innovaciones y que esas intensidades dependen de la empresa en particular que tiene competencias para identificarlas, lo que significa, que una innovación puede conducir a cambios significativos en la estructura de una empresa, mientras que otras, poco aportan en los procesos de adaptación al cambio. Por otro lado, según Simmie y Strambach (2006), las economías del mundo deben centrarse en productos y servicios que realmente les añadan valor a través de la innovación, ya que es a través de esta que se renovan los mercados y los procesos.

Según Gallouj et al. (2007), la innovación de servicios es sin duda, asimilada a la adopción e implementación de sistemas técnicos que surgen de los sectores industriales, en detrimento de otras formas de innovación algo menos tangibles. Los recursos invertidos en tecnología llevan a las empresas a una mayor eficacia operacional, haciendo que productos y servicios tengan mejor cualidad de acuerdo Gallaugher (2007), principalmente en los servicios, que es precisamente lo que las empresas de transporte público buscan.

\section{TECNOLOGIAS APLICADAS A LOS TRANSPORTES}

Hay muchas tecnologías de transporte desarrolladas por la humanidad. En este trabajo, se presentarán algunos tipos de estas tecnologías en las que se ha presentado innovaciones científicas aplicadas en la amplia área del transporte, y de la cual aún se espera que continúe siendo blanco de las novedades más evolucionadas, ya que muestra ejemplos de transporte sobre la base de criterios sustentables, que eviten el consumo de combustibles contaminantes no renovables.

Un vehículo eléctrico es movido por lo menos un motor eléctrico. Mientras que los vehículos con combustión interna pueden o no tener un motor eléctrico. Los motores eléctricos de los vehículos de combustión interna suelen estar vinculados a los sistemas periféricos, como el accionamiento eléctrico las ventanas.

\subsection{Vehículos elétricos}

Contrariamente a lo que muchos creen, la tecnología de los automóviles híbridos y eléctricos no es una innovación tecnológica reciente. Aunque sin duda hay importantes avances tecnológicos en los vehículos eléctricos de hoy en día, como baterías de iones de litio y toda la tecnología digital en los vehículos modernos, sin embargo, en esencia, el concepto básico permanece. En otras palabras, no hubo cambios radicales en los motores eléctricos de hoy en día, incluso cuando es utilizada la energía cinética generada por el movimiento del vehículo (Baran et al, 2010).

Según Rajashekar (1994), la historia del vehículo eléctrico se remonta a finales de los años 70 y comienzos de los 80 en el momento del embargo petrolero árabe, donde los precios de la gasolina dejaron de ser una preocupación para desarrollar vehículos eléctricos. Algunos trabajos iniciales se llevaron a cabo por la General Motors a finales de los años 80 y surgieron principalmente para resolver los problemas ambientales y de energía, como consecuencia del 
uso de los vehículos con motores de combustión interna (Hori et al., 1998).

\subsection{Ventajas del vehículo eléctrico}

El inminente agotamiento de las fuentes de energía primaria, la dependencia energética del petróleo y el impacto medioambiental conduce a la inversión en nuevos modelos de energía para la movilidad dirigidos a mejorar la calidad de vida de la población y la reducción de la factura energética mundial. El objetivo principal consiste en contribuir a la movilidad sostenible, maximizando las ventajas y la integración de las energías renovables como una alternativa a los combustibles fósiles (Ferry, 2013) y la aplicación y el uso del vehículo eléctrico.

Para Pompermayer (2010), los vehículos eléctricos son excelentes candidatos para reemplazar a los carros convencionales en su uso más frecuente, que incluye principalmente el trayecto diario de la casa al trabajo y vuelta a casa al final de la jornada. Los estudios realizados en Europa indican que alrededor del $80 \%$ de los europeos, en promedio, conducen menos de 40 kilómetros por día.

Para este tipo de distancias, el vehículo eléctrico es perfectamente aplicable ya que se trata de menores recorridos, permitidos por las baterías, que tienen baja autonomía y que convierten el VE en una de sus principales limitaciones. Además de la menor emisión de gases de efecto invernadero que puede ser hasta cero, dependiendo del método de generación de electricidad, los carros eléctricos no producen otros contaminantes como partículas, y son mucho más silenciosos que los vehículos convencionales.

En la actualidad, la "Ruta Europea de Transportes", de la Comisión Europea propone diez objetivos a alcanzar entre los próximos veinte y cuarenta años. Algunos objetivos de mayor relevancia incluyen la reducción de la contribución de los vehículos de combustión interna en el transporte urbano hasta del $50 \%$ en 2030 y su cambio gradual a los vehículos eléctricos en las ciudades para el año 2050 (Golinska, et. al., 2012).

Sin embargo, mientras que la primera generación de vehículos eléctricos que ya hace parte del mercado, todavía no es claro si el público aceptar ampliamente la movilidad eléctrica. Esta incertidumbre se genera debido principalmente a diversas cuestiones que aún están limitando el mercado de vehículos eléctricos y la gama relativamente limitada, además de problemas con las baterías (Gaines et al., 2011), la interfaz con la red eléctrica y el futuro crecimiento de los costos energía (Caín et al., 2010).

Por otra parte, Gómez (2010) afirma que los principales problemas asociados con los vehículos eléctricos son los costos adicionales relacionados al peso, tamaño y distribución de las baterías, además de la poca disponibilidad de puntos de recarga para este tipo de vehículos.

\subsection{Incentivos ao veículo elétrico}

Existen básicamente cinco tipos de acciones gubernamentales utilizadas en el mundo para fomentar la difusión de los vehículos eléctricos: bonificaciones a los compradores de vehículos eléctricos, descuentos en los impuestos, adopción de restricciones en el uso de los vehículos convencionales, apoyo a la investigación y despliegue en la infraestructura.

Este apoyo se ha reforzado recientemente cuando varios países han explotado paquetes de beneficios introducidos durante la crisis económica mundial para promover una discriminación en favor de los vehículos híbridos y eléctricos (De Castro, 2010). En Europa, 17 de los 28 países 
ya han adoptado diversas medidas a través de proyectos diseñados para fomentar el uso del voto eléctrico, como subsidios gubernamentales y algunas estrategias de ventas de los fabricantes.

En el caso de Portugal, por medio de la Resolución del Consejo de Ministros No 81/2009, se crearon 5.000 euros cada 5.000 becas para la compra de VE, el mismo importe de la subvención ha sido adoptada por otros países como Irlanda y Francia (Mapa Movilidad Tecnológico eléctrica, 2012). Ya en el Reino Unido a partir de enero de 2011, comprometió £43 millones para subvencionar la compra de vehículos eléctricos que van desde $£ 5.000$ hasta con el $25 \%$ del precio de compra del vehículo (Shepherd et al. 2012).

A pesar de que en Alemania la compra de vehículos no cuenta con subsidios económicos, en mayo de 2011 fueron aprobados otro tipo de incentivos como excepción de impuestos, cupos de parqueo y circulación especial de los vehículos eléctricos en áreas restringidas. (Mapa Tecnológico Movilidad Eléctrica, 2012).

La política energética actual de los EE.UU. subvenciona la compra de vehículos eléctricos con un crédito fiscal de hasta $\$ 7,500$ por vehículo dependiendo en parte del tamaño de la batería. Algunos estados complementan esta subvención. California, por ejemplo, agrega \$3.000 para un total de \$ 10.500 (Hidrue et al., 2011).

En Brasil, estas medidas están mucho más lejanas, siendo el comprador real el responsable de todos los costos de mantenimiento y la compra de su vehículo. Según De Castro, et al. (2010), los vehículos eléctricos no reciben un tratamiento diferenciado, aunque algunas medidas están en estudio. En el caso del Impuesto sobre Productos Industrializados (IPI), hasta el año 2015, los vehículos eléctricos se clasifican en la categoría "otros", al que se le atribuía un impuesto del $25 \%$.

En la actualidad, el apoyo del gobierno prácticamente restringida a la promoción de la investigación, sin embargo, un grupo interministerial integrado por representantes de los Ministerios de Finanzas, Desarrollo, Ciencia y Tecnología y el Medio Ambiente, así como representantes de la industria, ha analizado las bases de un plan para fomentar el vehículo eléctrico (De Castro, 2010).

En algunos estados de Brasil, hay una exoneración del cobro de impuestos a la propiedad (Impuesto sobre la Propiedad del Automotor) para vehículos eléctricos o motor eléctrico. La Tabla 1 muestra los estados en donde la categoría de vehículo eléctrico se encuentra exenta de la recaudación de impuestos de propiedad (Dos Santos et al., 2010).

\begin{tabular}{l|l}
\hline \multicolumn{1}{c|}{ ESTADO } & \multicolumn{1}{c}{ ARTIGO } \\
\hline Ceará & Lei 12.023 - art. 4, IX - vehículos movidos a motor eléctrico \\
\hline Maranhão & Lei 5.594 - art. 9, XI - vehículos movidos com fuerza motriz eléctrica \\
\hline Pernambuco & Lei 10.849 - art. 5, XI - vehículos movidos a motor eléctrico \\
\hline Piauí & Lei 4.548 - art. 5, VII - vehículos movidos a motor eléctrico \\
\hline Rio Grande do Norte & Lei 6.967 - art. 8, XI - vehículos movidos a motor eléctrico \\
\hline Rio Grande do Sul & Lei 8.115 - art. 4, II - vehículos movidos com fuerza motriz eléctrica \\
\hline Sergipe & Lei 3.287 - art. 4, XI - vehículos movidos a motor eléctrico \\
\hline
\end{tabular}

Tabla 1 - Estados que exoneran los vehículos eléctricos del cobro del IPVA

\section{METODOLOGIA}

La metodología adoptada en este estudio considera la investigación y las consultas sobre los sitios web oficiales de varios países, colecciones literarias, y análisis de la documentación 
pertinente para la cuestión que permitió un estudio de la evolución del uso de la propulsión eléctrica de vehículos y gasolina, con sus características y usos, así como su característica innovadora y sostenible antes de que los otros sistemas de propulsión.

El costo marginal se evaluó a través de las perspectivas económicas. Para ello, se plantearán los valores básicos relacionados con los costos de adquisición, mantenimiento e inspección durante un período $100.000 \mathrm{~km}$, la autonomía de cada uno de los vehículos, así como los impuestos sobre la propiedad y los valores seguros, así:

\subsection{Aplicación del método}

Para el estudio se seleccionaron los modelos de vehículos Gol Special 1.0 (movido a gasolina) y el Nissan Leaf (eléctrico). El modelo Gol fue elegido por pertenecer a una de las empresas de con mayores ventas nacionales y el Nissan Leaf por ser un vehículo que ya se está probando en Brasil, operando en la Policía Militar del Estado de Río de Janeiro. El precio de compra del Gol es R \$ 28.000,00 (VW, 2014) y el Nissan Leaf R \$ 90,000.00 (Nissan, 2014).

El costo por kilómetro de vehículo de gasolina se obtuvo por la inversa de la autonomía (15 $\mathrm{km} / \mathrm{l}$ ) y se multiplicó por el precio medio del combustible en el Distrito Federal para el año 2014 de R \$3,17 (ANP, 2014). El vehículo eléctrico tuvo un rango de $180 \mathrm{~km}$ para 4 horas de carga, por lo que el resultado de la división de las horas de carga para la autonomía en kilómetros es 0.02223 horas $/ \mathrm{km}$, para calcular el costo, fue multiplicado el factor encontrado por el precio de 0,30275 kw/h Distrito Federal para el año 2014.

El mantenimiento del vehículo de gasolina fue diseñado por la propia información de los fabricantes, los costos de revisión y mantenimiento prestados a los $100.000 \mathrm{~km}$ son iguales a $\mathrm{R} \$ 17,934$.

Fue identificado como el elemento más caro del vehículo eléctrico el mantenimiento del conjunto de baterías de US\$15.600 cada 100.000 kilómetros. Además otras revisiones por valor de $\mathrm{R} \$ 1,594.22$. La depreciación fue adoptada en $20 \%$, impuestos a la propiedad igual al $4 \%$ y el $5 \%$ de seguros para los dos tipos de vehículos, estos valores se centrarán en el precio de compra de cada vehículo.

\section{RESULTADOS}

Para saber el costo por kilómetro de cada vehículo, los factores analizados se convirtieron y se dividen por 100.000 kilómetros. Este valor se adoptó debido a la duración de la primera cambio de baterías de los vehículos eléctricos. En la Tabla 2 son mostrados los resultados.

\begin{tabular}{|c|ccccccc|c|}
\cline { 2 - 9 } \multicolumn{1}{c|}{} & Autonomia & Aquisição & Depreciação & Manutenção & IPVA & Seguro & Final \\
\hline VG & $\mathrm{R} \$ 0,2115$ & $\mathrm{R} \$ 0,2800$ & $\mathrm{R} \$ 0,0500$ & $\mathrm{R} \$ 0,1793$ & $\mathrm{R} \$ 0,0112$ & $\mathrm{R} \$ 0,0140$ & $\mathrm{R} \$ 0,7460$ \\
\hline $\mathrm{VE}$ & $\mathrm{R} \$ 0,0067$ & $\mathrm{R} \$ 0,9000$ & $\mathrm{R} \$ 0,1800$ & $\mathrm{R} \$ 0,4184$ & $\mathrm{R} \$ 0,0360$ & $\mathrm{R} \$ 0,0450$ & $\mathrm{R} \$ 1,5861$ \\
\hline
\end{tabular}

Tabla 5.1 - Costo en Reales por km, para $100.000 \mathrm{~km}$ recorridos

Se observa que el único elemento en el que VE es más rentable es la autonomía, además de ser el más destacado entre todos los componentes evaluados en ambos casos. En comparación con el costo de comprar un vehículo eléctrico es también el más alto entre todos los elementos estudiados. La figura 1 permite ver las diferencias observadas el precio de cada ítem analizado. 


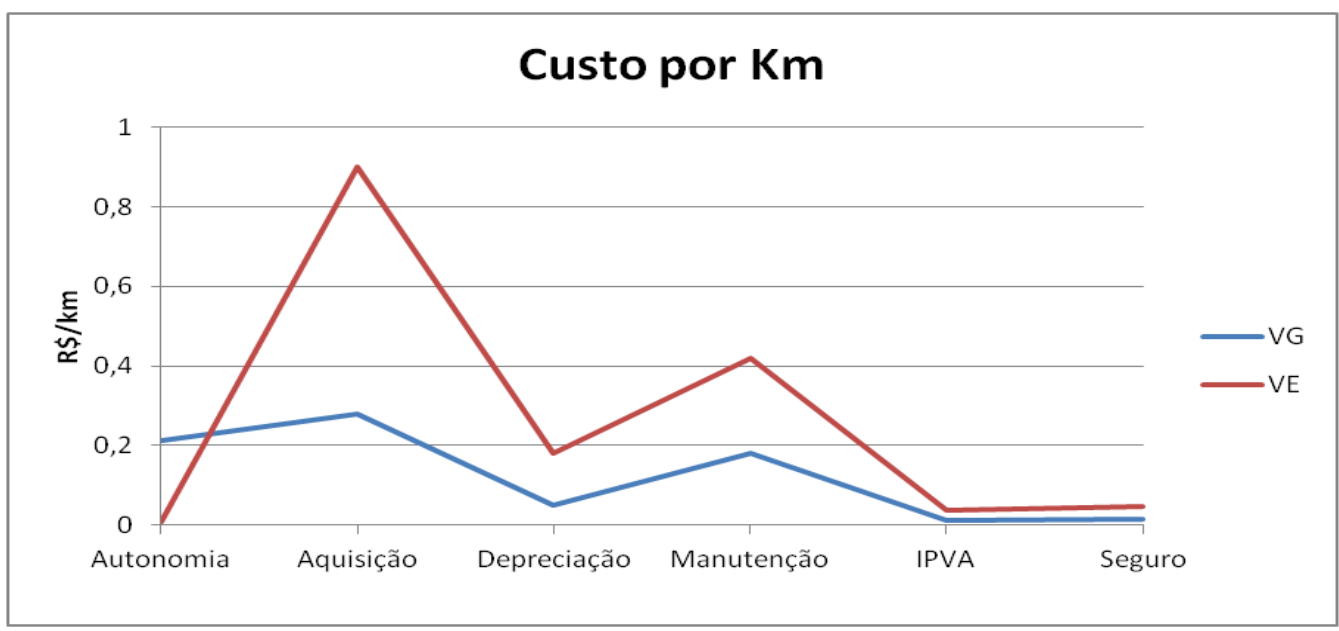

Figura 5.1 - Costo por kilometro para cada tipo de vehíuclo

\section{DISCUSIONES Y CONSIDERACIONES FINALES}

Los resultados obtenidos demuestran la realidad del vehículo eléctrico en Brasil. Su alto costo aún está lejos de las condiciones de un vehículo más limpio. La comparación entre la gran inserción del vehículo eléctrico en el mercado europeo y el mercado brasileño es inevitable, pero el volumen de kilómetros recorridos entre los dos lugares es también hace la diferencia. En Brasil, el carro tiene un uso mucho más intenso que en Europa, lo que sin duda acorta el tiempo de sustitución de la batería.

La diferencia en el coste final del 55,35\% entre los vehículos demuestra la dificultad del mercado para enfrentar el vehículo eléctrico en Brasil. En Europa y en algunos países existe un incentivo a la compra de vehículos como bajas tasas de estacionamiento y cuotas anuales, sin embargo, el manteniendo todavía demuestra ser costosa. Sin embargo, el VE también se convierte en una alternativa interesante para los grandes beneficios estratégicos y ambientales nacionales.

Otra diferencia en el caso de Brasil y otros países en relación con el fomento de un vehículo limpio es la fuerza motriz de cada modelo. Brasil, además de contar una fuerte industria de petróleo, otorga subsidios a los combustibles, a diferencia de otros que se atribuyen este tipo de subsidios a los vehículos. Para Brasil aumentar su flota de vehículos eléctricos, los incentivos fiscales deben actuar en dos zonas, la adquisición y el mantenimiento (especialmente las baterías). Si Brasil no adopta incentivos que otros países otorgan, el VE seguirá manteniéndose alejado de poder adquisitivo de los brasileros.

La autonomía fue el único indicador de los cinco estudiados en este trabajo representa un atractivo al momento de pensar en la compra de un vehículo eléctrico, lo que limita las oportunidades para para que este tipo de tecnologías entren en el mercado brasileño.

\section{Referencias}

ANEEL. Agência Nacional de Energia Elétrica. Disponível em: <www.aneel.gov.br/area> Acesso em: 23 de novembro de 2014.

ANP. Agência Nacional de Petróleo, Gás Natural e Biocombustível. Fonte: Disponível em: <www.anp.gov.br/preco/prc/Resumo_Por_Estado_Municipio.asp> Acesso em: 23 de novembro de 2014. ANP. Agência nacional do Petróleo, Gás natural e Biocombustível. Site oficial.

BALSA, Joana Maria Rocha. "Avaliação do impacto da introdução de veículos elétricos na procura de combustiveis em Portugal." (2013). 
CIT2016 - XII Congreso de Ingeniería del Transporte València, Universitat Politècnica de València, 2016. DOI: http://dx.doi.org/10.4995/CIT2016.2016.3408

BARAN, Renato, and Luiz Fernando Loureiro Legey. "Veículos elétricos: história e perspectivas no Brasil." XIII Congresso Brasileiro de Energia, Brasil. 2010.

BATTERY University. Disponível em: <batteryuniversity.com/learn/article/electric_vehicle> Acesso em: 23 de novembro de 2014.

BECKER, Thomas A., Ikhlaq Sidhu, and Burghardt Tenderich. "Electric vehicles in the United States: A new model with forecasts to 2030." Center for Entrepreneurship and Technology, University of California, Berkeley 2009.1 (2009).

CAIN, Anna, Iain MacGill, and Anna Bruce. "Assessing the potential impacts of electric vehicles on the electricity distribution network'." AuSES Annual Conference, Canberra, Australia, accessed. Vol. 3. 2013.

DE CASTRO, Bernardo Hauch Ribeiro, and Tiago Toledo Ferreira. "Veículos elétricos: aspectos básicos, perspectivas e oportunidades." BNDES Setorial 32 (2010): 267-310.

DE GENNARO, Michele, et al. "A pilot study to address the travel behaviour and the usability of electric vehicles in two Italian provinces." Case Studies on Transport Policy (2014).

DE LA ENERGÍA, Observatorio Tecnológico. "Mapa Tecnológico Movilidad Eléctrica." Ministerio de Industria, Energía y Turismo (2012).

DIJK, Marc, Renato J. Orsato, and René Kemp. "The emergence of an electric mobility trajectory." Energy Policy 52 (2013): 135-145.

Disponível em: <exame.abril.com.br/seu-dinheiro/noticias/manter-carro-por-10-anos-custa-menos-do-quetrocar-de-carro>. Acesso em: 23 de novembro de 2014.

Disponível em: <www.eco-cars.net/electriccarforsale.php?selproid=237> Acesso em: 23 de novembro de 2014.

Disponível em: <www.nissan.pt/PT/pt/tool/car-builder/leaf.html> Acesso em: 23 de novembro de 2014.

Disponível em: 〈www.vw.com.br/pt.html> Acesso em: 23 de novembro de 2014.

DURAND, T. Strategizing for innovation: competence analysis in assessing strategy change. In: HEENE, A; SANCHEZ, R. Competence-based strategic management. Chichester, England, p.127-150, 1997.

GAINES, L., et al. "Life-cycle analysis for lithium-ion battery production and recycling." Transportation Research Board 90th Annual Meeting, Washington, DC. 2011.

GALLAUGHER, John M. Strategic positioning and resource- based thinking: cutting through the haze of punditry to understand factors behind sustainable, successful internet businesses. International Journal of EBusiness Research, v. 3, n. 3, p. 14-25, 2007.

GALLOUJ, Faïz \& SANSON, Katia. Economia da inovação: um balanço dos debates recentes. In: BERNARDES, Roberto \& ANDREASSI, Tales. Inovação em serviços intensivos em conhecimento. São Paulo: Saraiva, p. 3-27. 2007.

GOLINSKA, Paulina, and Marcin Hajdul. "European Union Policy for Sustainable Transport System: Challenges and Limitations." Sustainable Transport. Springer Berlin Heidelberg, 2012. 3-19.

GOMES, Luís Manuel Martins. "O Veículo Eléctrico e a sua Integração no Sistema Eléctrico." Instituto Superior Técnico-Universidade Técnica de Lisboa. Disponível em: https://dspace. ist. utl. pt/bitstream/2295/767651/1/dissertacaoLuisGomes. pdf. Acedido (2010).

GREMAUD, A.P.; TONETO, R.R. \& VASCONCELOS, M.A.S. Economia brasileira contemporânea. 5 ed., São Paulo: Atlas, 2004.

HIDRUE, Michael K., et al. "Willingness to pay for electric vehicles and their attributes." Resource and Energy Economics 33.3 (2011): 686-705.

HORI, Yoichi, Yasushi Toyoda, and Yoshimasa Tsuruoka. "Traction control of electric vehicle: Basic experimental results using the test EV "UOT Electric March"." Industry Applications, IEEE Transactions on 34.5 (1998): 1131-1138.

LOUNSBURY, M. \& CRUMLEY, T. E. New practice creation: an institutional perspective on innovation. In: Organizational Studies. Sage publications, 2007.

ÔNIBUS ELÉTRICO HÍBRIDO: uma esperança pelos novos fios da tecnologia. Canal do Ônibus.

POMPERMAYER, Fabiano Mezadre. "Etanol e veículos elétricos: via de mão única ou dupla?." Cadernos Fórum Nacional 10 (2010).

RAJASHEKARA, Kaushik. "History of electric vehicles in General Motors. " IEEE transactions on industry applications 30.4 (1994): 897-904.

RASKIN, Amy, and Saurin Shah. The Emergence of Hybrid Vehicles: Ending Oil's Stranglehold on Transportation and the Economy. AllianceBernstein, 2006.

Resolução do Conselho de Ministros n. 81/2009. Diário da República, $1^{a}$ série, $n^{o} 173$ - 7 de Fevereiro, 2009 (http://www.apve.pt/upload/docs/rcm81_2009.pdf).

SHEPHERD, Simon, Peter Bonsall, and Gillian Harrison. "Factors affecting future demand for electric vehicles: A model based study." Transport Policy 20 (2012): 62-74.

SIMMIE, James \& STRAMBACH, Simone. The contribution of KIBS to innovation in cities: an evolutionary and institutional perspective. Journalof Knowledge Management, v. 10, n. 5; p. 26-40, September, 2006. 